\title{
Intramolecularly Sensitized Precipitons: A Model System for Application to Metal Sequestration
}

\author{
Mark R. Ams and Craig S. Wilcox* \\ Department of Chemistry and The Combinatorial Chemistry Center, University of Pittsburgh,
}

Pittsburgh, Pennsylvania 15260

\section{Supporting Information}

\section{Table of Contents}

Page

General Experimental

Experimental Procedures

${ }^{1} \mathrm{H}$ and ${ }^{13} \mathrm{C}$ spectra of compounds

General Information: $\quad{ }^{1} \mathrm{H}$ NMR spectra were recorded on a Bruker Avance 300 at $300 \mathrm{MHz} .{ }^{13} \mathrm{C}$ NMR spectra were record on a Bruker Avance 300 at $75 \mathrm{MHz}$ or a Bruker Avance 600 at $150 \mathrm{MHz}$. The chemical shifts are given in parts per million $(\mathrm{ppm})$ on the delta scale $(\delta)$, and the coupling constant values $(J)$ are in hertz. The solvent peak was used as the reference value. For ${ }^{1} \mathrm{H} \mathrm{NMR}: \mathrm{CDCl}_{3}=7.27$ ppm; $\mathrm{CD}_{2} \mathrm{Cl}_{2}=5.32 ; \mathrm{CD}_{3} \mathrm{CN}=1.94$. For ${ }^{13} \mathrm{C} \mathrm{NMR}: \mathrm{CDCl}_{3}=77.23 \mathrm{ppm} ; \mathrm{CD}_{2} \mathrm{Cl}_{2}=54.00 ; \mathrm{CD}_{3} \mathrm{CN}=$ 1.39. Abbreviations for NMR data: $\mathrm{s}=$ singlet; $\mathrm{d}=$ doublet; $\mathrm{t}=$ triplet; $\mathrm{q}=$ quartet; $\mathrm{dd}=$ doublet of doublets; $\mathrm{dt}=$ doublet of triples; $\mathrm{dq}=$ doublet of quartets; $\mathrm{tt}=$ triplet of triplets; $\mathrm{m}=$ multiplet; $\mathrm{br}=$ broad; app = apparent, $\mathrm{ABq}=\mathrm{AB}$ quartet.

High resolution and low resolution mass spectra were recorded of a VG 7070 spectrometer. Infrared (IR) spectra were collected on an Avatar 380 Nicolet FT-IR spectrometer. Samples for IR were prepared either as a thin film on a $\mathrm{NaCl}$ plate by dissolving the sample in $\mathrm{CH}_{2} \mathrm{Cl}_{2}$ and then evaporating the $\mathrm{CH}_{2} \mathrm{Cl}_{2}$ or as a $\mathrm{KBr}$ pellet. Melting points were determined using a Thomas Hoover capillary melting point apparatus and are uncorrected. UV-Vis absorption spectra were obtained in degassed solvent at $23{ }^{\circ} \mathrm{C}$ using an Aligent 8453 spectrophotometer. Emission spectra were recorded in degassed solvent solution at $23{ }^{\circ} \mathrm{C}$ with a Cary Eclipse fluorescence spectrometer equipped with Varian software.

Thin layer chromatography (TLC) was performed using E. Merck silica gel 60F-254 (0.25 mm) analytical glass plates. Light absorption by compounds was observed using ultraviolet light $(254 \mathrm{~nm}$ or $365 \mathrm{~nm}$ ). Silica gel columns for flash chromatography, according to the method of Still, were prepared with E. Merck silica gel 60 (230-240 mesh ASTM).

Dry solvents were distilled shortly before use from an appropriate drying agent under nitrogen atmosphere. Tetrahydrofuran (THF), diethyl ether $\left(\mathrm{Et}_{2} \mathrm{O}\right)$, and toluene were distilled from sodium and benzophenone. Dry methanol was distilled from magnesium turnings and stored under nitrogen or was purchased as anhydrous and used as obtained. Ethyl acetate was dried over $4 \AA$ molecular sieves for at least $24 \mathrm{~h}$ prior to use. N,N-Dimethylformamide (DMF) was dried over $\mathrm{MgSO}_{4}$, distilled in vacuo, and stored over $4 \AA$ molecular sieves. Pyridine was distilled from calcium hydride and stored over $4 \AA$ molecular sieves. Hexane refers to the mixed hydrocarbon fraction (bp 68-70 ${ }^{\circ} \mathrm{C}$ ), principally $n$-hexane, and was purified as follows: the commercial solvent was stirred over concentrated $\mathrm{H}_{2} \mathrm{SO}_{4}$ for at least 24 h, decanted, stirred over anhydrous $\mathrm{NaHCO}_{3}$ for at least $6 \mathrm{~h}$, decanted, and distilled. Benzene, 
methylene chloride $\left(\mathrm{CH}_{2} \mathrm{Cl}_{2}\right)$ and triethylamine (TEA) were distilled from $\mathrm{CaH}_{2}$. Anhydrous dimethylsulfoxide (DMSO) and acetonitrile $\left(\mathrm{CH}_{3} \mathrm{CN}\right)$ were purchased from Acros and used as is. Other commercially available reagents and solvents were reagent grade and were used without further purification.

Reactions performed under a nitrogen atmosphere were arranged with a mercury bubbler so that the system could be alternately evacuated and filled with nitrogen and left under positive pressure. Syringes and reaction flasks were dried in an oven at $120{ }^{\circ} \mathrm{C}$ and cooled in a desiccator over calcium sulphate prior to use. Reactions at "room temperature" were conducted under ambient laboratory conditions $\mathrm{T}=$ $20-27{ }^{\circ} \mathrm{C}, \mathrm{p}=720-770 \mathrm{mmHg}$. References to "removal of volatile components in vacuo" refer to rotary evaporation of the sample at $25-65{ }^{\circ} \mathrm{C}$ in vacuo $(18-25 \mathrm{mmHg})$, followed by removal of residual volatile materials under vacuum $(0.05-0.5 \mathrm{mmHg})$ at room temperature.

\section{EXPERIMENTAL PROCEDURES}<smiles>BrCc1ccc(-c2ccccn2)nc1</smiles>

5-Bromomethyl-2,2'-bipyridine ${ }^{1}$. 5-Methyl-2,2'-bipyridine (1.99 g, $\left.11.8 \mathrm{mmol}\right)$, $\mathrm{N}$-bromosuccinimide $(2.09 \mathrm{~g}, 11.8 \mathrm{mmol}$ ), and azobis(iso-butyronitrile) (483 $\mathrm{mg}, 2.94 \mathrm{mmol})$ were dissolved in $\mathrm{CCl}_{4}(179 \mathrm{~mL})$ under $\mathrm{N}_{2}$ and the mixture was stirred at an internal solvent temperature of $43{ }^{\circ} \mathrm{C}$ for $23 \mathrm{~h}$. Reaction progress was monitored by ${ }^{1} \mathrm{H}$ NMR to observe the consumption of NBS and the formation of product. After $23 \mathrm{~h}$, an additional $0.5 \mathrm{eq}$ of NBS was added and the mixture was stirred at $43{ }^{\circ} \mathrm{C}$ for 2 additional $\mathrm{h}$. The mixture was cooled to $23{ }^{\circ} \mathrm{C}$, filtered, and the volatile components of the filtrate were removed in vacuo to afford a crude solid that was purified by flash chromatography $\left(\mathrm{SiO}_{2}, 5: 1 \mathrm{Hex}:\right.$ EtOAc, column was pretreated with 19:1 Hex: $\mathrm{NEt}_{3}$ then washed with 5:1 Hex:EtOAc) to afford 5-Bromomethyl-2,2'-bipyridine as a white solid (1.35 g, 46\%): $\mathrm{R}_{f} 0.15$ (5:1 Hex:EtOAc, pretreated with 19:1 Hex:NEt $)$; mp 74-76 ${ }^{\circ} \mathrm{C}$; IR (KBr) 3050, 2998, 2967, 1596, 1556, 1461, 1434, 1392, 1252, 1203, 1127, 1088, 1039, 834, 797, 749, 730, 650; The ${ }^{1} \mathrm{H}$ and ${ }^{13} \mathrm{C}$ NMR data matches the data described by Ballardini and coworkers ${ }^{1}:{ }^{1} \mathrm{H}$ NMR $\left(300 \mathrm{MHz}, \mathrm{CDCl}_{3}\right) \delta 8.70($ app d, J $=2 \mathrm{~Hz}, 2 \mathrm{H}), 8.43(\mathrm{dd}, \mathrm{J}=6,2 \mathrm{~Hz}, 2 \mathrm{H}), 7.89-7.81(\mathrm{~m}, 2 \mathrm{H}), 7.34$ (ddd, J = 7, 5, $1 \mathrm{~Hz}, 1 \mathrm{H}), 4.55$ (s, 2 $\mathrm{H}) ;{ }^{13} \mathrm{C}$ NMR $\left(75 \mathrm{MHz}, \mathrm{CD}_{3} \mathrm{CN}\right) \delta 156.3,155.8,149.6,149.5,137.8,137.2,133.9,124.2,121.5$, 121.3, 29.9.

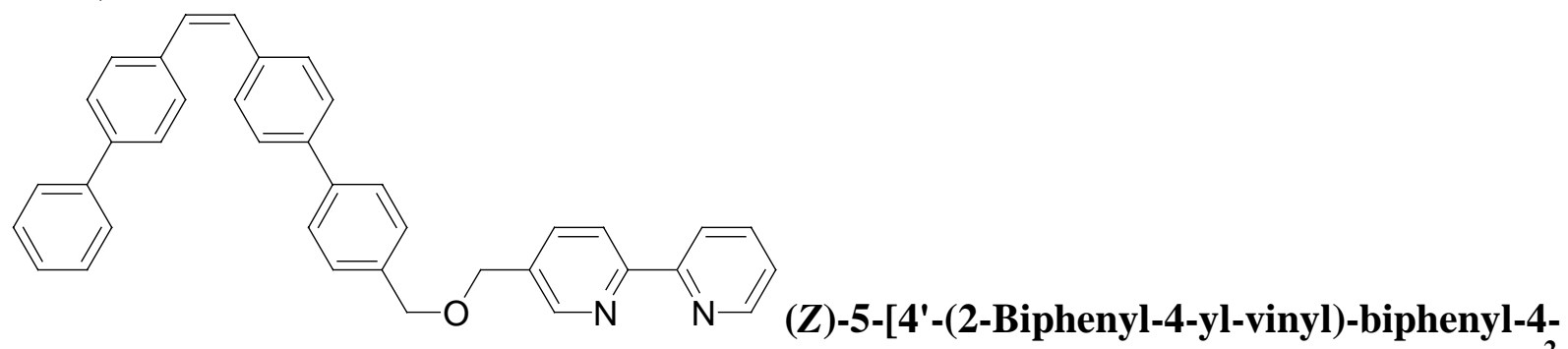

ylmethoxymethyl]-[2,2']bipyridine (4Z). To a cooled solution $\left(0^{\circ} \mathrm{C}\right)$ of benzyl alcohol $3 Z^{2}(500 \mathrm{mg}$,

\footnotetext{
${ }^{1}$ Ballardini, R.; Balzani, V.; Clemente-Leon, M.; Credi, A.; Gandolfi, M.; Ishow, E.; Perkins, J.; Stoddart, F.; Tseng, H.; Wenger, S. J. Am. Chem. Soc. 2002, 124, 12786.
}

2 (a) Bosanac, T. Ph. D. Dissertation, University of Pittsburgh, 2003. (b) Bosanac, T.; Wilcox, C. S. J. Am. Chem. Soc. 2002, 124, 4194. (c) Honigfort, M.; Brittain, W. J.; Bosanac, T.; Wilcox, C. S. Macromolecules 2002, $35,4849$. 
$1.38 \mathrm{mmol})$ in THF $(138 \mathrm{~mL})$ was added dry $\mathrm{NaH}(66.2 \mathrm{mg}, 2.76 \mathrm{mmol})$. The solution was warmed to $23{ }^{\circ} \mathrm{C}$ and stirred for $2 \mathrm{~h}$. 5-Bromomethyl-2,2'-bipyridine ( $354 \mathrm{mg}, 1.42 \mathrm{mmol}$ ) was then added, stirred for $2 \mathrm{~h}$ at $23{ }^{\circ} \mathrm{C}$, then heated at reflux for $17.5 \mathrm{~h}$. The solution was cooled to $23{ }^{\circ} \mathrm{C}$ and volatile components of the reaction mixture were removed in vacuo. The crude solid was combined with $\mathrm{H}_{2} \mathrm{O}$ $(300 \mathrm{~mL})$ and the solution was extracted with $\mathrm{CH}_{2} \mathrm{Cl}_{2}(3 \times 20 \mathrm{~mL})$. The combined organic phases were washed with brine $(360 \mathrm{~mL})$, dried with $\mathrm{MgSO}_{4}$, and filtered. Volatile components in the filtrate were removed in vacuo to afford a crude solid that was purified by flash chromatography $\left(\mathrm{SiO}_{2}, \mathrm{CH}_{2} \mathrm{Cl}_{2}\right.$ then 99:1 $\left.\mathrm{CH}_{2} \mathrm{Cl}_{2}: \mathrm{MeOH}\right)$ to afford $\mathbf{4 Z}$ as a pale green solid (537 mg, 73\%): $\mathrm{R}_{f} 0.31\left(\mathrm{CH}_{2} \mathrm{Cl}_{2}\right) ; \mathrm{mp} \mathrm{113-115}$ ${ }^{\circ} \mathrm{C}$; IR (KBr) 3030, 3003, 2857, 1598, 1588, 1557, 1460,1395, 1353, 1088, 881, 815; UV-Vis $\left(\mathrm{CH}_{3} \mathrm{CN}\right.$, $10 \mu$ M) $\lambda_{\max } 274 \mathrm{~nm}, \varepsilon 34200 \mathrm{M}^{-1} \mathrm{~cm}^{-1}, 290 \mathrm{~nm}, \varepsilon 33200 \mathrm{M}^{-1} \mathrm{~cm}^{-1}, 330 \mathrm{~nm}, \varepsilon 24800 \mathrm{M}^{-1} \mathrm{~cm}^{-1}$; ${ }^{1} \mathrm{H}$ NMR $\left(300 \mathrm{MHz}, \mathrm{CD}_{3} \mathrm{CN}\right) \delta 8.69-8.66(\mathrm{~m}, 2 \mathrm{H}), 8.43-8.41(\mathrm{app} \mathrm{d}, \mathrm{J}=8 \mathrm{~Hz}, 2 \mathrm{H})$, 7.95-7.90 (m, $\left.2 \mathrm{H}\right), 7.72-$ $7.63(\mathrm{~m}, 5 \mathrm{H}), 7.56(\operatorname{app~d}, \mathrm{J}=7 \mathrm{~Hz}, 4 \mathrm{H}), 7.46(\operatorname{app~d}, \mathrm{J}=8.5 \mathrm{~Hz}, 4 \mathrm{H}), 7.42(\operatorname{app~s}, 1 \mathrm{H}), 7.34(\operatorname{app~d}, \mathrm{J}$ $=8 \mathrm{~Hz}, 4 \mathrm{H}), 6.72(\mathrm{~s}, 2 \mathrm{H}), 4.67(\mathrm{~s}, 2 \mathrm{H}), 4.65(\mathrm{~s}, 2 \mathrm{H}) ;{ }^{13} \mathrm{C} \mathrm{NMR}\left(75 \mathrm{MHz}, \mathrm{CD}_{3} \mathrm{CN}\right) \delta 156.1,156.8$, $149.3,148.8,140.8,140.4,140.0,139.6,137.0,136.6,136.5,136.4,130.2,130.1,129.6,129.5,129.8$, $128.4,127.4,127.1,127.0,126.9,124.0,121.2,121.0,72.3,69.6 ; \mathrm{HRMS}(\mathrm{EI}) \mathrm{m} / z$ calcd for $\mathrm{C}_{38} \mathrm{H}_{30} \mathrm{~N}_{2} \mathrm{O}$ 530.2358 , found 530.2383 .

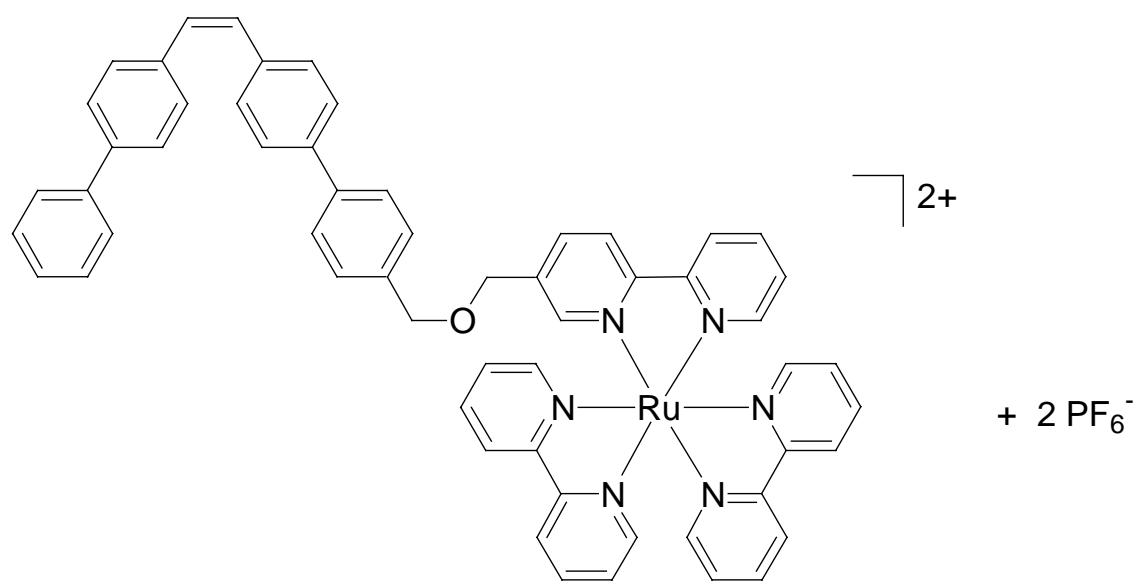

(Z)-[5-(4'-(2-Biphenyl-4-yl-vinyl)-biphenyl-4-ylmethoxymethyl]-[2,2']bipyridine]-bis(2,2'-

bipyridine)ruthenium(II)- bis(hexafluorophosphate) (2Z). A solution of cis-dichlorobis(2,2'bipyridine)ruthenium(II)dihydrate $(90.8 \mathrm{mg}, 0.188 \mathrm{mmol})$ and silver hexafluoroantimonate(V) $(132 \mathrm{mg}$, $0.376 \mathrm{mmol})$ in acetone $(12.5 \mathrm{~mL})$ under $\mathrm{N}_{2}$ was heated at reflux for $48 \mathrm{~h}$, followed by filtration of $\mathrm{AgCl}$. Bipyridine $4 Z(99.8 \mathrm{mg}, 0.188 \mathrm{mmol})$ was added to the filtrate and the mixture was heated at reflux for $24 \mathrm{~h}$. Volatile components of the reaction mixture were removed in vacuo to afford a crude red solid that was purified by flash chromatography $\left(\mathrm{SiO}_{2}, 19: 1 \mathrm{CH}_{3} \mathrm{CN}: 0.4 \mathrm{M} \mathrm{KNO}_{3}(\mathrm{aq})\right)$. The desired fractions were combined and volatile components were reduced in volume to $25 \mathrm{~mL}$. The solution was combined with $0.25 \mathrm{M} \mathrm{NH}_{4} \mathrm{PF}_{6}(10 \mathrm{~mL})$, and then stirred at $23{ }^{\circ} \mathrm{C}$ for $20 \mathrm{~min}$, and then extracted with $\mathrm{CHCl}_{3}(3 \times 100 \mathrm{~mL})$. The organic extract was washed with $\mathrm{H}_{2} \mathrm{O}(2 \times 200 \mathrm{~mL})$. Volatile components of the organic layer were removed in vacuo to afford complex $2 Z$ as a red solid (114 $\mathrm{mg}, 49 \%): \mathrm{R}_{f} 0.16$ (19:1 $\mathrm{CH}_{3} \mathrm{CN}: 0.4 \mathrm{M} \mathrm{KNO}_{3}$ (aq) on pretreated silica); mp 164-166 ${ }^{\circ} \mathrm{C}$; IR (KBr) 3027, 2919, 2853, 1603 , 1464, 1446, 1093, 838, 761, 730, 699; UV-Vis $\left(\mathrm{CH}_{3} \mathrm{CN}, 10 \mu \mathrm{M}\right) \lambda_{\max } 290 \mathrm{~nm}, \varepsilon 91800 \mathrm{M}^{-1} \mathrm{~cm}^{-1}, 340$ $\mathrm{nm}, \varepsilon 22900 \mathrm{M}^{-1} \mathrm{~cm}^{-1}, 450 \mathrm{~nm}, \varepsilon 13700 \mathrm{M}^{-1} \mathrm{~cm}^{-1}$; ${ }^{1} \mathrm{H}$ NMR $\left(300 \mathrm{MHz}, \mathrm{CD}_{3} \mathrm{CN}\right) \delta 8.49-8.46(\mathrm{~m}, 4 \mathrm{H})$, 8.44-8.39 (m, $2 \mathrm{H}$ ), 8.06-8.01 (app t, J = 15, $8 \mathrm{~Hz}, 4 \mathrm{H}$ ), 7.97 (dd, J = 5, $2 \mathrm{~Hz}, 1 \mathrm{H}), 7.92$ (dd, J = 8, 1 Hz, $1 \mathrm{H})$, 7.73-7.69 (m, $5 \mathrm{H})$, 7.65-7.60 (m, $6 \mathrm{H}), 7.58-7.55(\mathrm{~m}, 3 \mathrm{H}), 7.47-7.45(\mathrm{~m}, 2 \mathrm{H}), 7.42-7.34(\mathrm{~m}, 9$ H), 7.33-7.27 (m, $1 \mathrm{H}), 7.23(\mathrm{app} \mathrm{d}, \mathrm{J}=8,2 \mathrm{~Hz}, 2 \mathrm{H}), 6.74(\mathrm{~s}, 2 \mathrm{H}), 4.51(\mathrm{ABq}, \mathrm{J}=14 \mathrm{~Hz}, 2 \mathrm{H}), 4.47(\mathrm{~s}$, $2 \mathrm{H}) ;{ }^{13} \mathrm{C}$ NMR $\left(75 \mathrm{MHz}, \mathrm{CD}_{3} \mathrm{CN}\right) \delta 158.5,158.4,158.3,157.4,153.1,153.0,150.9,141.7,141.2$, $141.0,140.8,140.6,139.3,139.2,138.7,138.1,138.0,137.7,131.5,131.4,130.9,130.8,130.3,129.8$, $129.7,129.6,129.07,129.04,129.01,128.97,128.94,128.90,128.90,128.2,128.1,125.7,125.6,125.5$, 
125.3, 73.6, 69.6; HRMS(ES) $\mathrm{m} / z$ calcd for $\mathrm{C}_{58} \mathrm{H}_{46} \mathrm{~F}_{6} \mathrm{~N}_{6} \mathrm{OPRu}\left[\mathrm{M}-\mathrm{PF}_{6}\right]^{+}$1089.2418, found 1089.2452, $944.14\left[\mathrm{M}-2 \mathrm{PF}_{6}\right]^{+}$.

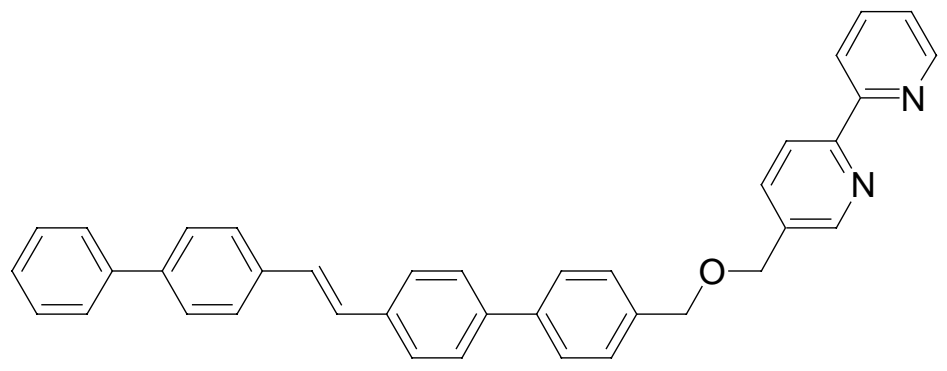

(E)-5-[4'-(2-Biphenyl-4-yl-vinyl)-biphenyl-4-ylmethoxymethyl]-[2,2']bipyridine (4E).

A solution of bipyridine $4 Z(153 \mathrm{mg}, 0.289 \mathrm{mmol})$ and diphenyl diselenide $(9.1 \mathrm{mg}, 0.029 \mathrm{mmol})$ in THF $(1.18 \mathrm{~mL})$ was heated at reflux for $23 \mathrm{~h}$. The solution was cooled to $23{ }^{\circ} \mathrm{C}$ and volatile components of the reaction mixture were removed in vacuo. The crude solid was triturated with $\mathrm{Et}_{2} \mathrm{O}(3$ x $5 \mathrm{~mL}$ ), filtered, and the volatile components of the filtrate were removed in vacuo to afford ligand $\mathbf{4 E}$ as a yellow solid $(45.5 \mathrm{mg}, 84 \%)$ : $\mathrm{mp} \mathrm{300-310}{ }^{\circ} \mathrm{C}$; $\mathrm{IR}(\mathrm{KBr}) 3048,3030,2855,1911,1589,1557$, $1496,1461,1436,1397,1357,1081,970,832,807,795,763,752,742,731,691 ;{ }^{1} \mathrm{H}$ NMR $(300 \mathrm{MHz}$, $\left.\mathrm{CDCl}_{3}\right) \delta$ 8.75-8.69 (m, $\left.2 \mathrm{H}\right), 8.46(\operatorname{app~d}, \mathrm{J}=8 \mathrm{~Hz}, 2 \mathrm{H}), 7.90(\mathrm{dd}, \mathrm{J}=8,2 \mathrm{~Hz}, 1 \mathrm{H}), 7.85(\mathrm{dd}, \mathrm{J}=8,2$ $\mathrm{Hz}, 1 \mathrm{H}), 7.66-7.62(\mathrm{~m}, 12 \mathrm{H}), 7.50$ (app d, J = $9 \mathrm{~Hz}, 3 \mathrm{H}$ ), 7.43 (app s, $1 \mathrm{H}), 7.38-7.34$ (m, $2 \mathrm{H}), 7.20$ (s, $2 \mathrm{H}), 4.68(\mathrm{~s}, 2 \mathrm{H}), 4.67(\mathrm{~s}, 2 \mathrm{H}) ;{ }^{13} \mathrm{C} \mathrm{NMR}$, too insoluble to obtain; HRMS(EI) $\mathrm{m} / \mathrm{z}$ calcd for $\mathrm{C}_{38} \mathrm{H}_{31} \mathrm{~N}_{2} \mathrm{O}[\mathrm{M}+\mathrm{H}]$ 531.2436, found 531.2452.

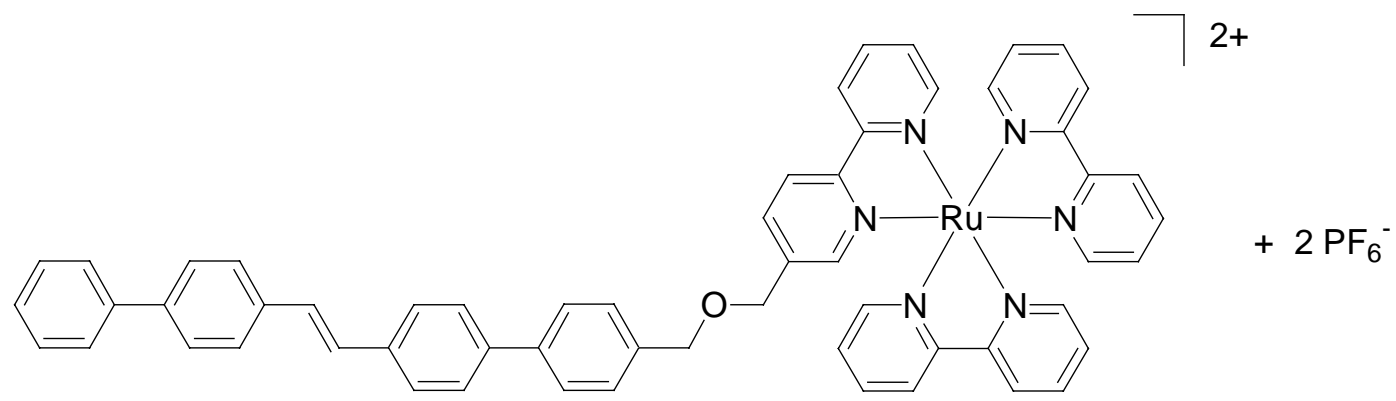

(E)-[5-(4'-(2-Biphenyl-4-yl-vinyl)-biphenyl-4-ylmethoxymethyl]-[2,2']bipyridine]-bis(2,2'-

bipyridine)ruthenium(II)-bis(hexafluorophosphate) (2E). A solution of cis-dichlorobis(2,2'bipyridine)ruthenium(II)dihydrate $(75.6 \mathrm{mg}, 0.156 \mathrm{mmol})$ and silver hexafluoroantimonate(V) $(107 \mathrm{mg}$, $0.312 \mathrm{mmol}$ ) in acetone $(10.4 \mathrm{~mL})$ under $\mathrm{N}_{2}$ was heated at reflux for $48 \mathrm{~h}$, followed by filtration of $\mathrm{AgCl}$. Bipyridine $\mathbf{4 E}(82.8 \mathrm{mg}, 0.156 \mathrm{mmol})$ was added to the filtrate and the mixture was heated at reflux for $22.5 \mathrm{~h}$. Volatile components of the reaction mixture were removed in vacuo to afford a crude red solid that was purified by flash chromatography $\left(\mathrm{SiO}_{2}, 19: 1 \mathrm{CH}_{3} \mathrm{CN}: 0.4 \mathrm{M} \mathrm{KNO}_{3}(\mathrm{aq})\right)$. The desired fractions were combined and volatile components were reduced in volume to $25 \mathrm{~mL}$. To the solution was added $0.25 \mathrm{M} \mathrm{NH}_{4} \mathrm{PF}_{6}(10 \mathrm{~mL})$ and then stirred at $23{ }^{\circ} \mathrm{C}$ for 20 min. The solution was then extracted into $\mathrm{CHCl}_{3}(3 \times 100 \mathrm{~mL})$ and washed with $\mathrm{H}_{2} \mathrm{O}(2 \times 200 \mathrm{~mL})$. Volatile components of the filtrate were removed in vacuo to give a red residue that was recrystallized from $\mathrm{CHCl}_{3} / \mathrm{Et}_{2} \mathrm{O}$ and then $\mathrm{CH}_{2} \mathrm{Cl}_{2} / \mathrm{EtOH}$ to afford a red solid (3.6 mg, 5\%): $\mathrm{R}_{f} 0.84\left(19: 1 \mathrm{CH}_{3} \mathrm{CN}: 0.4 \mathrm{M} \mathrm{KNO}_{3}\right.$ (aq) on pretreated silica); UV-Vis $\left(\mathrm{CH}_{3} \mathrm{CN}, 10 \mu \mathrm{M}\right) \lambda_{\max } 290 \mathrm{~nm}, \varepsilon 51500 \mathrm{M}^{-1} \mathrm{~cm}^{-1}, 339 \mathrm{~nm}, \varepsilon 28700 \mathrm{M}^{-1} \mathrm{~cm}^{-1}, 456 \mathrm{~nm}, \varepsilon$ $7400 \mathrm{M}^{-1} \mathrm{~cm}^{-1}$; ${ }^{1} \mathrm{H}$ NMR (300 MHz, $\left.\mathrm{CD}_{3} \mathrm{CN}\right)$ 8.50-8.42 (m, $\left.6 \mathrm{H}\right), 8.07-7.99(\mathrm{~m}, 4 \mathrm{H})$, 7.99-7.93 (m, 2 H), 7.80-7.60 (m, $16 \mathrm{H})$, 7.57-7.54 (m, $1 \mathrm{H})$, 7.54-7.44 (m, $2 \mathrm{H}), 7.44-7.30(\mathrm{~m}, 9 \mathrm{H}), 7.26(\mathrm{~d}, \mathrm{~J}=8 \mathrm{~Hz}, 2$ H), 4.58-4.50 (m, $2 \mathrm{H}), 4.50-4.44(\mathrm{~m}, 2 \mathrm{H}) ;{ }^{13} \mathrm{C} \mathrm{NMR}\left(151 \mathrm{MHz}, \mathrm{CD}_{3} \mathrm{CN}\right) \delta$ 157.97, 157.94, 157.90, $156.9,152.72,152.66,152.63,150.4,141.26,141.12,140.82,140.49,140.31,138.80,138.79,138.17$, $137.85,137.59,137.17,129.95,129.28,129.22,129.04,128.60,128.56,128.49,128.42,128.25,128.22$, 
128.09, 128.04, 127.70, 125.25, 125.13, 124.85, 73.09, 69.12; HRMS(ES) $\mathrm{m} / z$ calcd for $\mathrm{C}_{58} \mathrm{H}_{46} \mathrm{~F}_{6} \mathrm{~N}_{6} \mathrm{OPRu}\left[\mathrm{M}-\mathrm{PF}_{6}\right]^{+}$1089.2418, found 1089.2368.
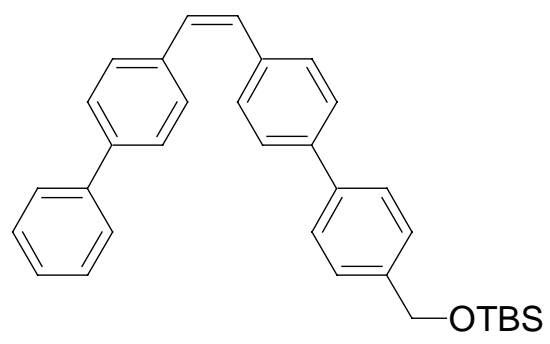

(Z)-[4'-(2-Biphenyl-4-yl-vinyl)-biphenyl-4-ylmethoxy]-tert-butyl-

dimethyl-silane ${ }^{3}(1 Z)$. To a solution of benzyl alcohol $3 Z(1.81 \mathrm{~g}, 5.0 \mathrm{mmol})$ in $\mathrm{CH}_{2} \mathrm{Cl}_{2}(10 \mathrm{~mL})$ at 23 ${ }^{\circ} \mathrm{C}$ was added $\mathrm{TBSCl}(830 \mathrm{mg}, 5.5 \mathrm{mmol})$, followed by imidazole $(375 \mathrm{mg}, 5.5 \mathrm{mmol})$. The solution was stirred at $23{ }^{\circ} \mathrm{C}$ for $1 \mathrm{~h}$ then diluted with $\mathrm{Et}_{2} \mathrm{O}(50 \mathrm{~mL})$ and filtered. The filtrate was washed with $1 \mathrm{M} \mathrm{HCl}(25 \mathrm{~mL})$, sat aq $\mathrm{NaHCO}_{3}(3 \times 25 \mathrm{~mL})$, dried with $\mathrm{MgSO}_{4}$, and filtered. Volatile components of the filtrate were removed in vacuo to afford a residue that was purified by flash chromatography $\left(\mathrm{SiO}_{2}\right.$ 9:1 Hex:EtOAc) to afford TBS ether $\mathbf{Z} \boldsymbol{Z}$ as a white solid (2.09 g, 88\%): $\mathrm{R}_{f} 0.50$ (19:1 Hex:EtOAc); $\mathrm{mp}$ $69-70{ }^{\circ} \mathrm{C}$; IR (neat) 2926, 2851, 1487, 1252, 1057, 1004, 836, $775 \mathrm{~cm}^{-1} ;{ }^{1} \mathrm{H}$ NMR $\left(300 \mathrm{MHz}, \mathrm{CDCl}_{3}\right) \delta$ 7.63-7.56 (m, $4 \mathrm{H}), 7.52-7.31(\mathrm{~m}, 13 \mathrm{H}), 6.66(\mathrm{~s}, 2 \mathrm{H}), 4.79(\mathrm{~s}, 2 \mathrm{H}), 0.96(\mathrm{~s}, 9 \mathrm{H}), 0.12(\mathrm{~s}, 6 \mathrm{H}) ;{ }^{13} \mathrm{C}$ NMR (75 MHz, CDCl3) $\delta 140.8,140.0,139.9,139.5,136.5,136.4,130.2,130.1,129.6,129.0,127.5$, 127.1, 127.0, 126.7, 64.5, 26.2, 18.6, -0.5; HRMS m/e calcd for $\mathrm{C}_{33} \mathrm{H}_{36} \mathrm{OSi} 476.2535$, found 476.2512.

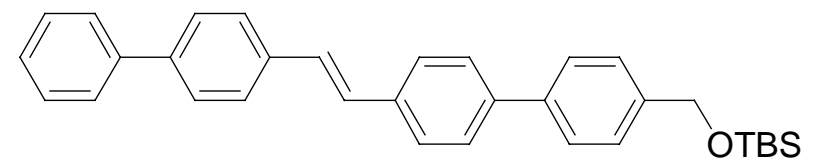

(E)-[4'-(2-Biphenyl-4-yl-vinyl)-biphenyl-4ylmethoxy]-tert-butyl-dimethyl-silane (1E). A solution of TBS ether $\mathbf{1 Z}(175 \mathrm{mg}, 368 \mu \mathrm{mol})$ and diphenyl diselenide $(12 \mathrm{mg}, 37 \mu \mathrm{mol})$ in THF $(1.5 \mathrm{ml})$ was heated at reflux for $4 \mathrm{~h}$. The solution was cooled to $23{ }^{\circ} \mathrm{C}$ and volatile components of the reaction mixture were removed in vacuo. The crude solid was triturated with $\mathrm{Et}_{2} \mathrm{O}(4 \mathrm{ml})$, filtered and washed with $\mathrm{Et}_{2} \mathrm{O}(2 \times 2 \mathrm{~mL})$. The volatile components of the filtrate were removed in vacuo and crude solid was triturates with hexanes $(3 \times 3$ $\mathrm{mL}$ ) and filtered. Solids were collected to afford TBS ether $\mathbf{1 E}$ as a white crystalline solid (93 $\mathrm{mg}, 53$ \%). $\mathrm{R}_{\mathrm{f}} 0.44$ (9:1 Hex:EtOAC); mp $260{ }^{\circ} \mathrm{C}$; IR (KBr) 2949, 2927, 2854, 1495, 1471, 1462, 1408, 1378 , 1252, 1089, 1062, 973, 835, $\mathrm{cm}^{-1}$; UV-Vis $\left(\mathrm{CH}_{2} \mathrm{Cl}_{2}, 10 \mu \mathrm{M}\right) \lambda_{\max } 340 \mathrm{~nm}, \varepsilon 58560 \mathrm{M}^{-1} \mathrm{~cm}^{-1}$; ${ }^{1} \mathrm{H}$ NMR $\left(300 \mathrm{MHz}, \mathrm{CDCl}_{3}\right) \delta$ 7.66-7.60 (m, $\left.12 \mathrm{H}\right), 7.49-7.33(\mathrm{~m}, 5 \mathrm{H}), 7.20(\mathrm{~s}, 2 \mathrm{H}), 4.81(\mathrm{~s}, 2 \mathrm{H}), 0.98(\mathrm{~s}, 9 \mathrm{H})$, $0.14(\mathrm{~s}, 6 \mathrm{H}) ;{ }^{13} \mathrm{C} \mathrm{NMR}$, too insoluble to obtain; HRMS m/e calcd for $\mathrm{C}_{33} \mathrm{H}_{36} \mathrm{OSi} 476.2535$, found 476.2515 .

\footnotetext{
${ }^{3}$ Bosanac, T.; Wilcox, C. S. Org. Lett. 2004, 6, 3221.
} 
$\operatorname{OD} \angle 9)^{\circ}$

$\angle 9 E 09^{\circ} \textrm{ }$ OL269. I690L $L$

О๑६ВГ ट-

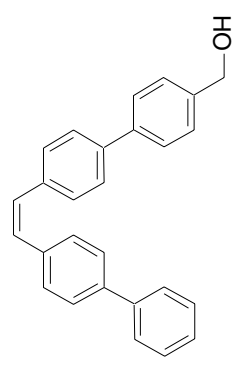

프

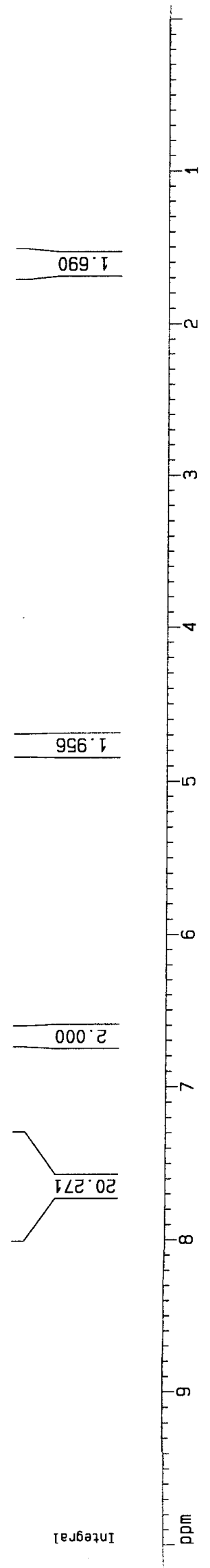




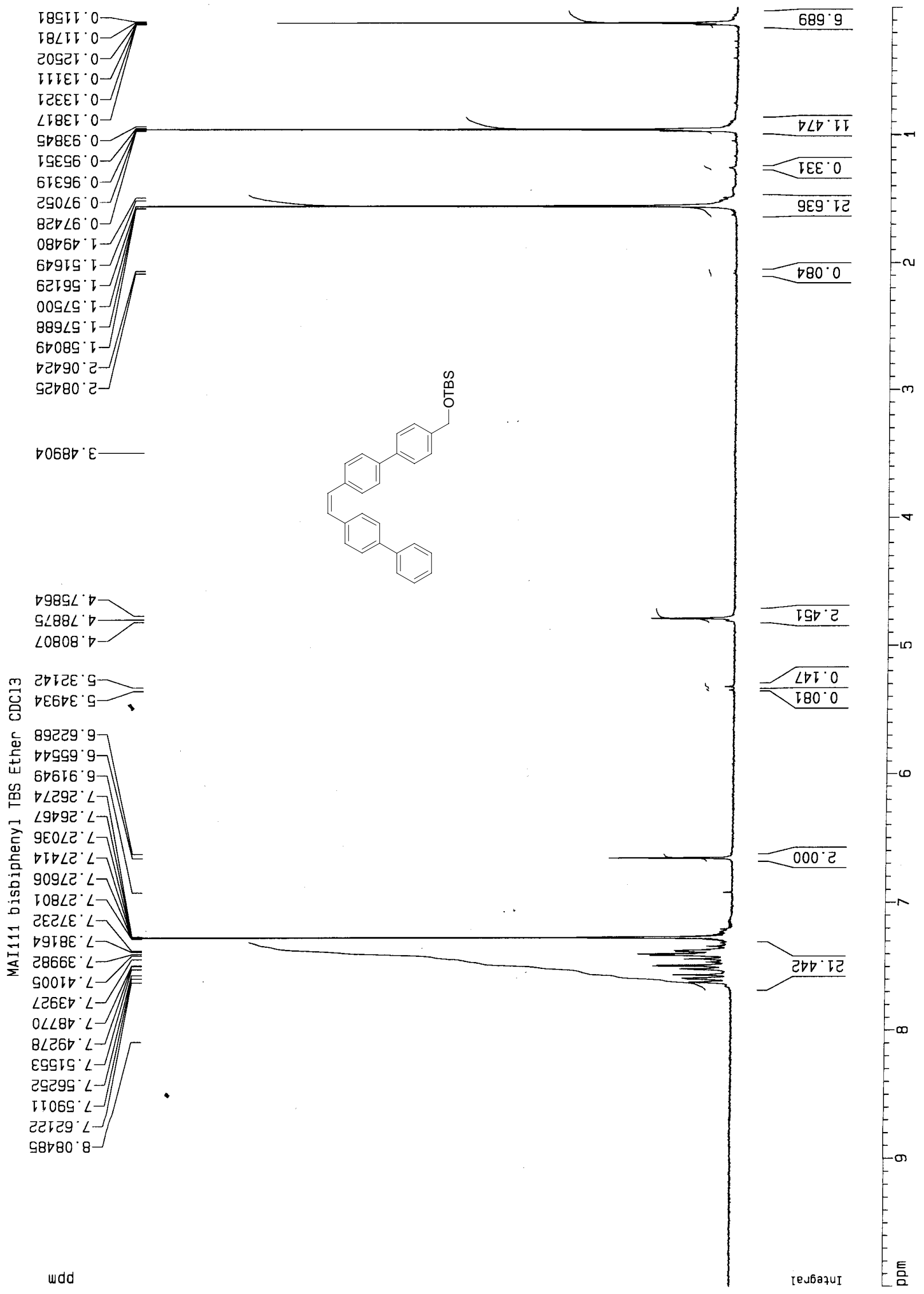


$2860^{\circ}$

$\forall \nabla 9^{\circ} 8$

SOL' 92

$296{ }^{*} \nabla 9$

$808 \cdot 9 L$

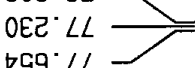

$\nabla 99^{\circ} \angle L$
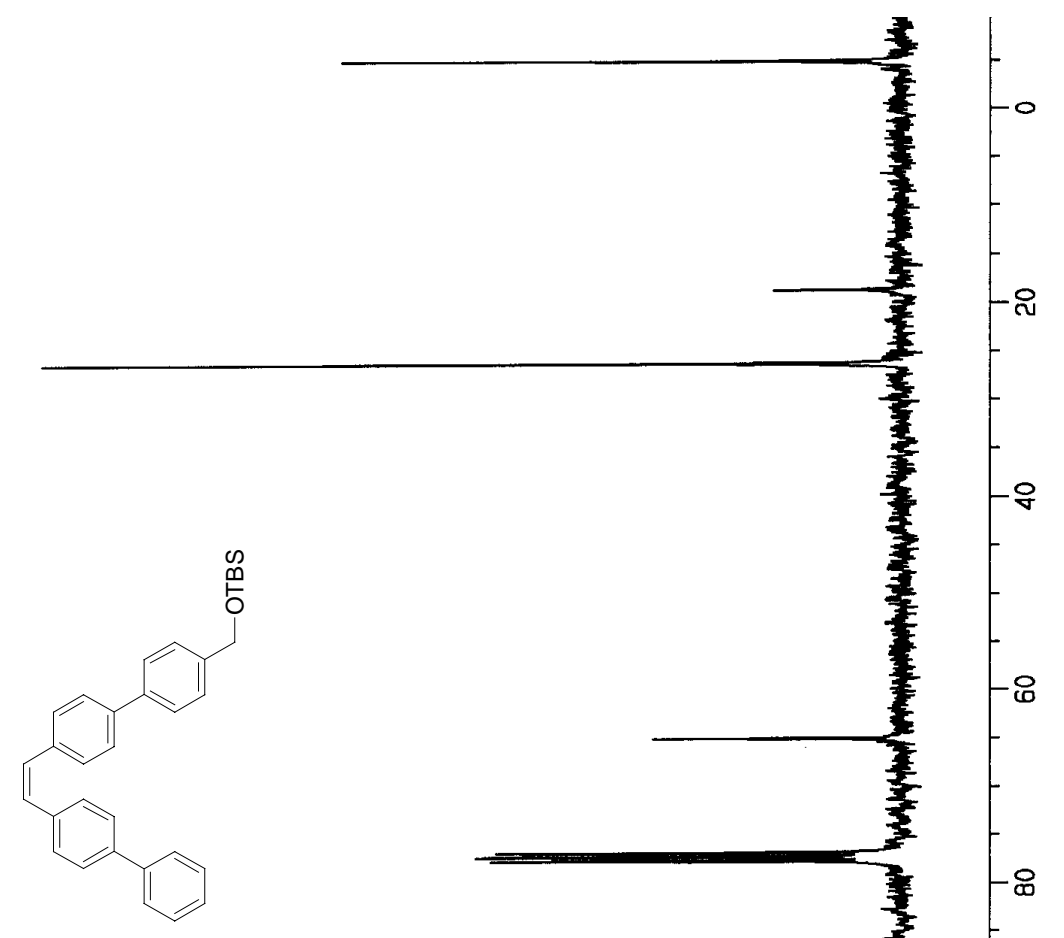

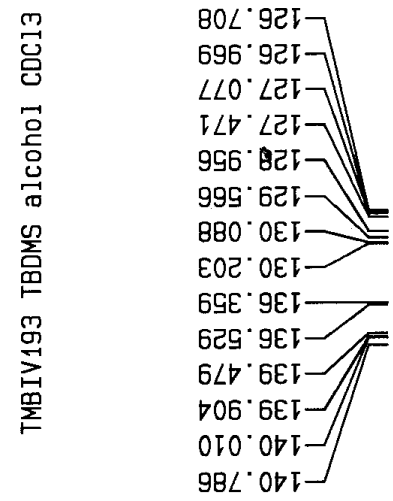

(1)
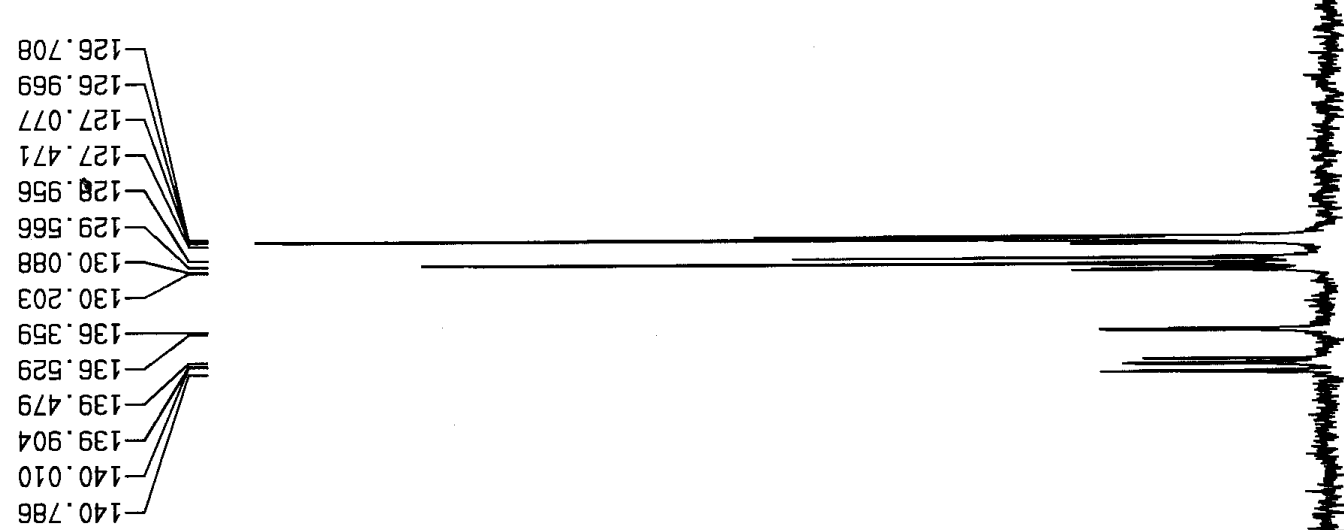

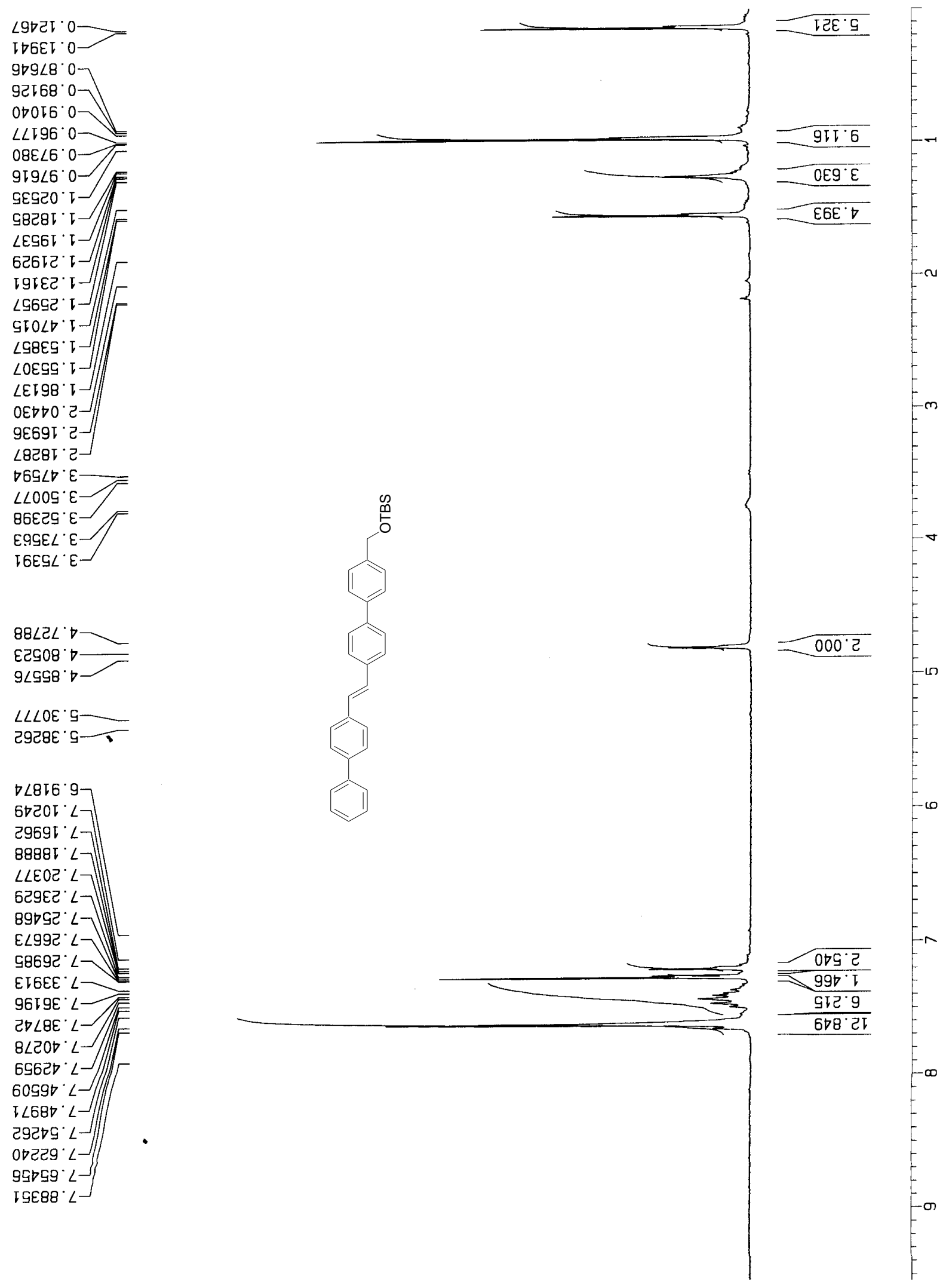

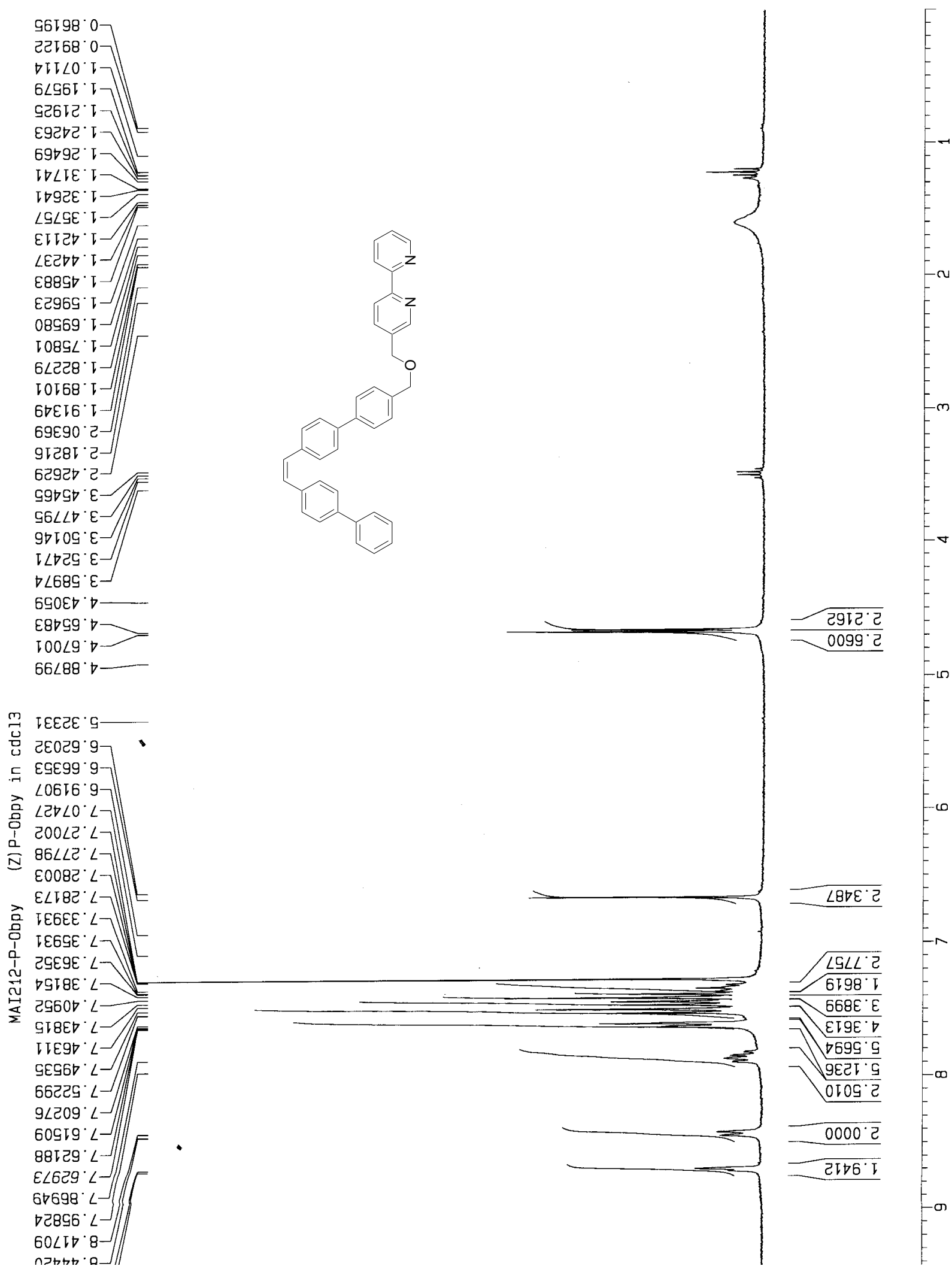

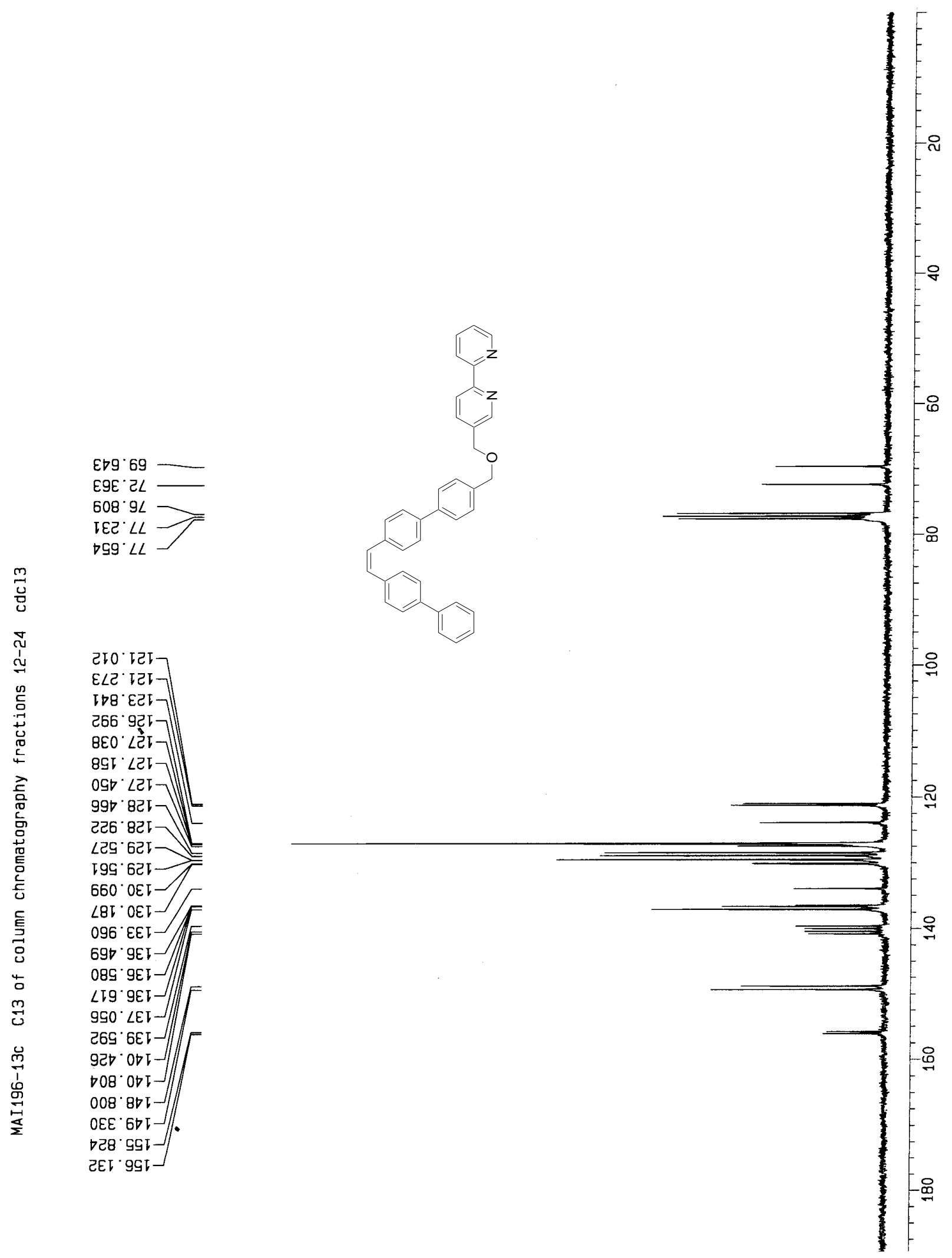

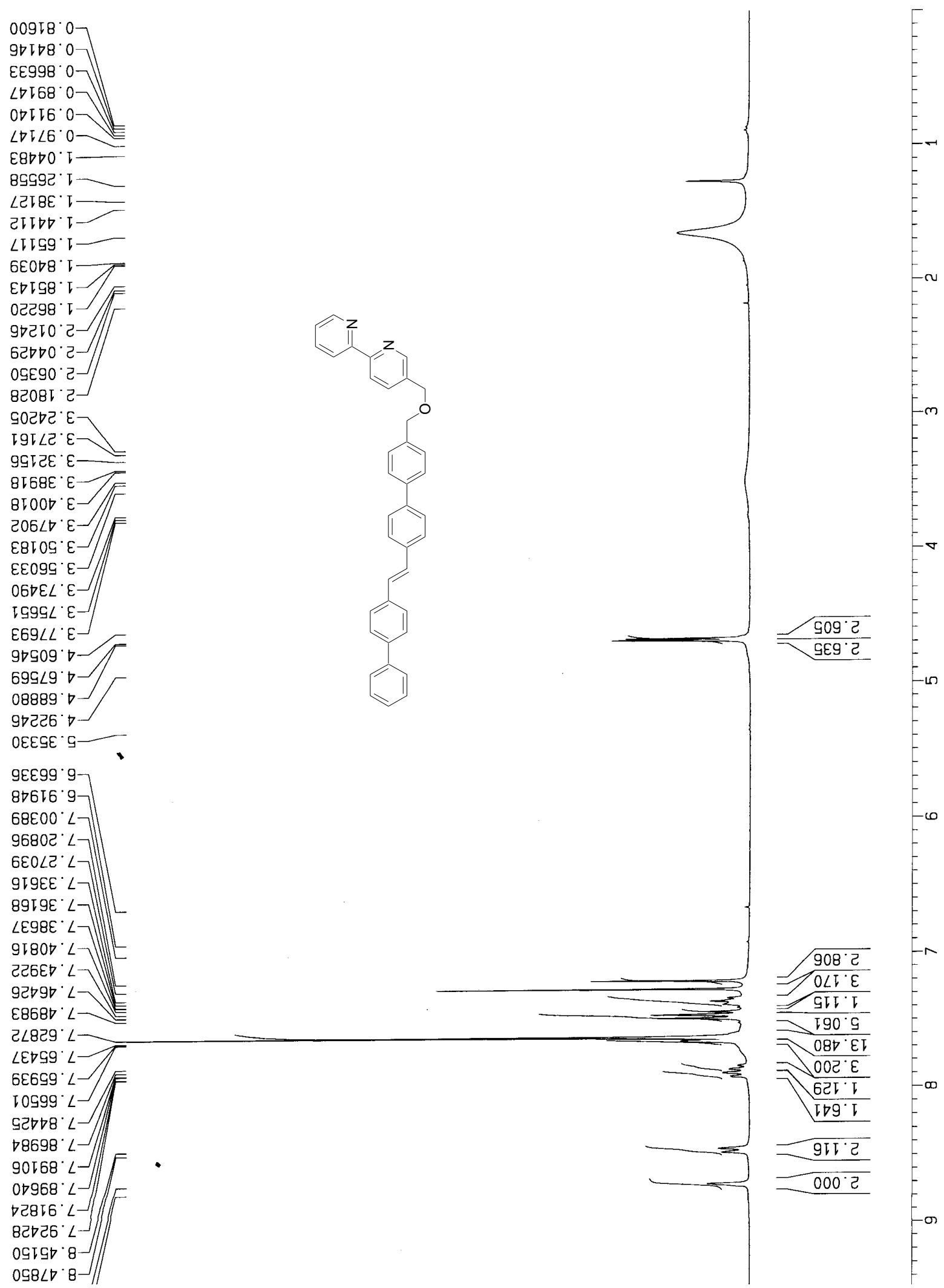


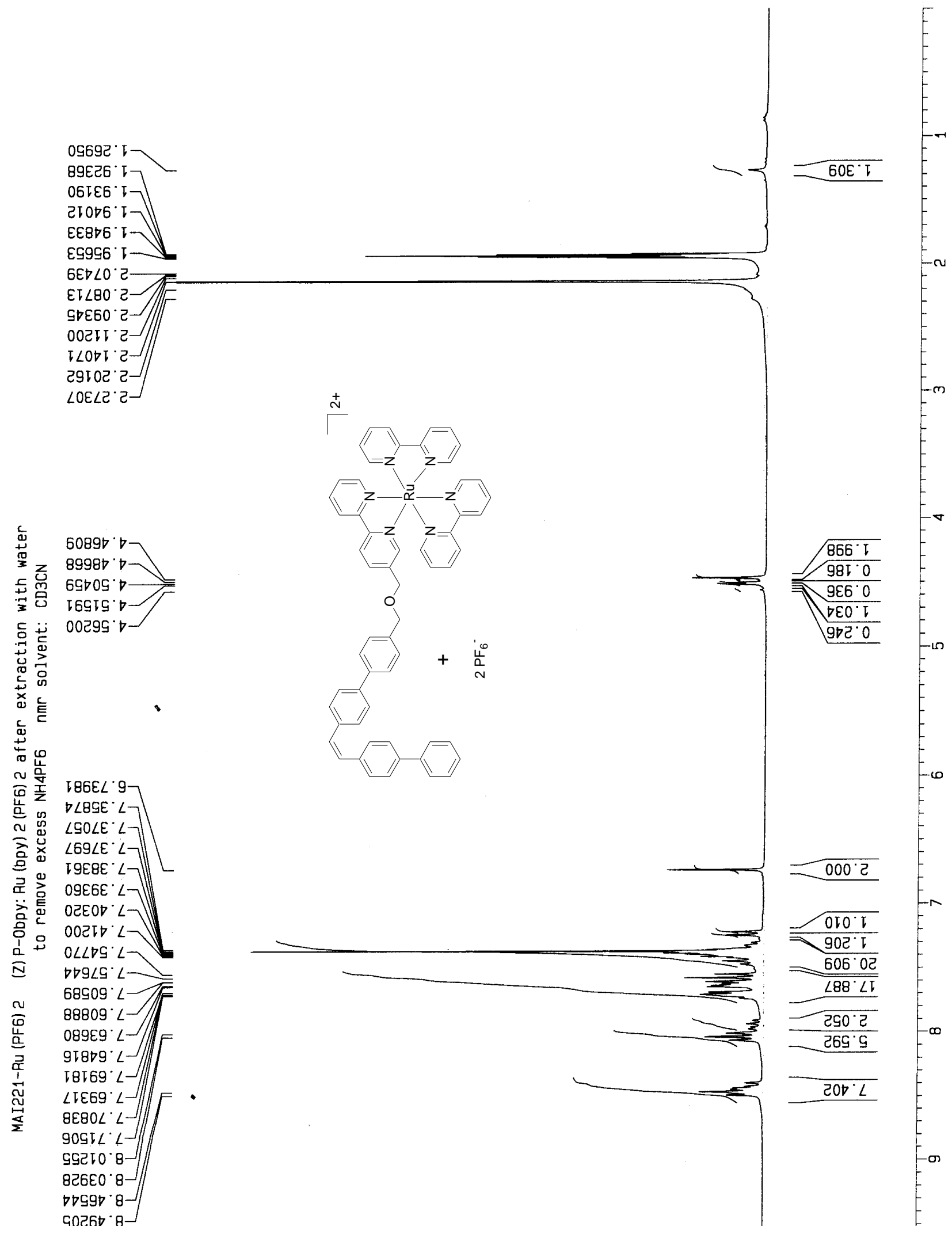




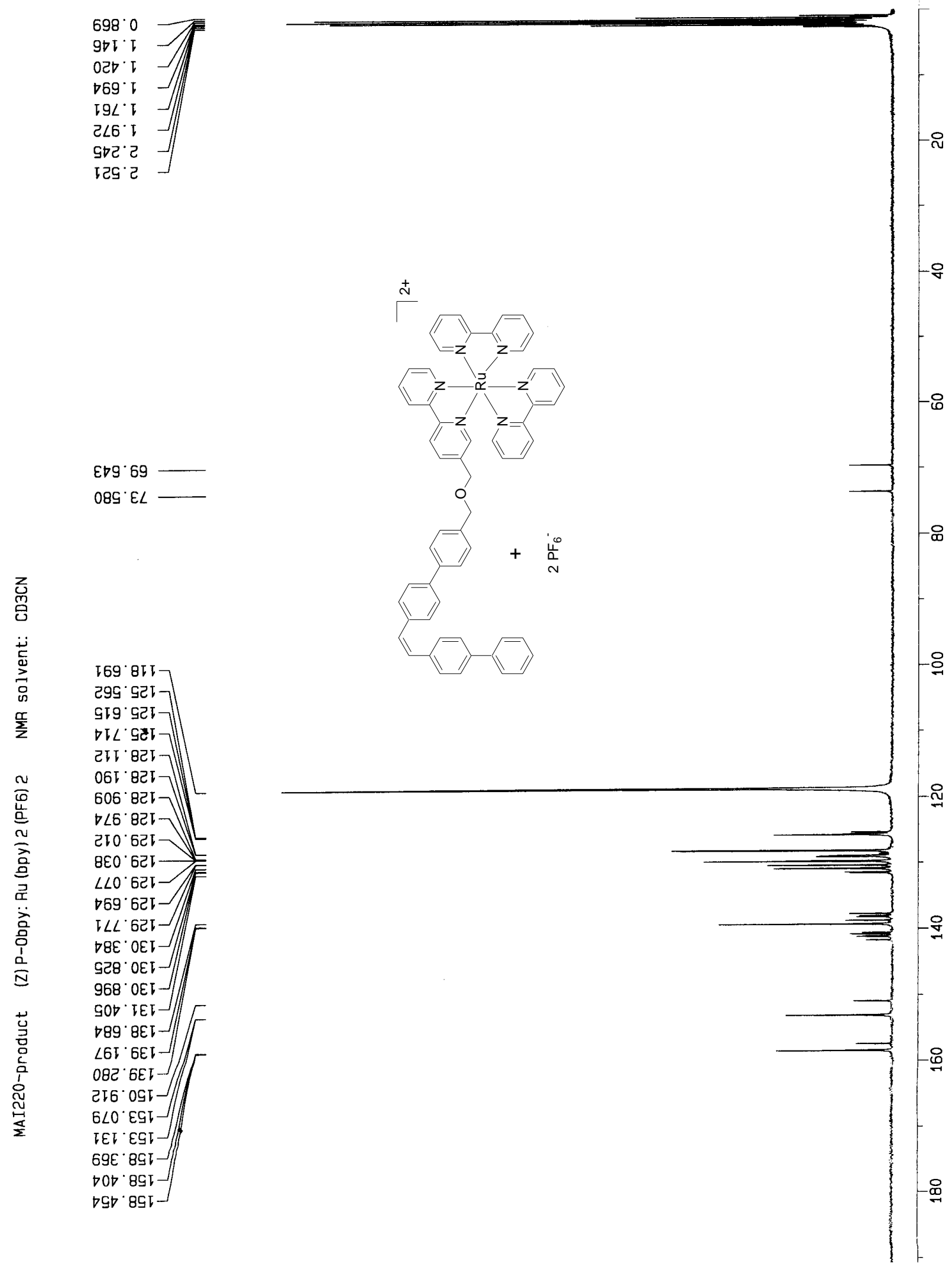




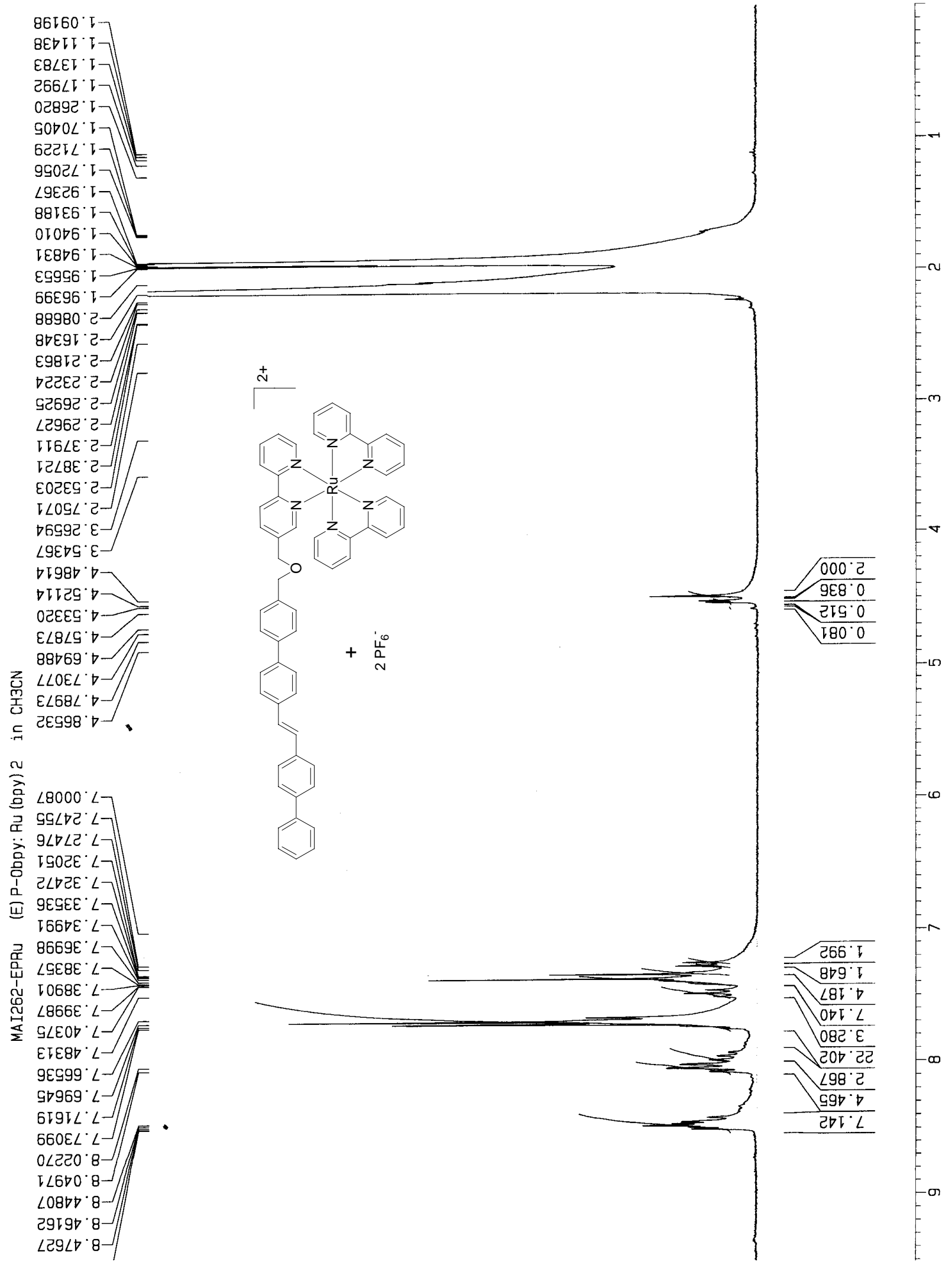




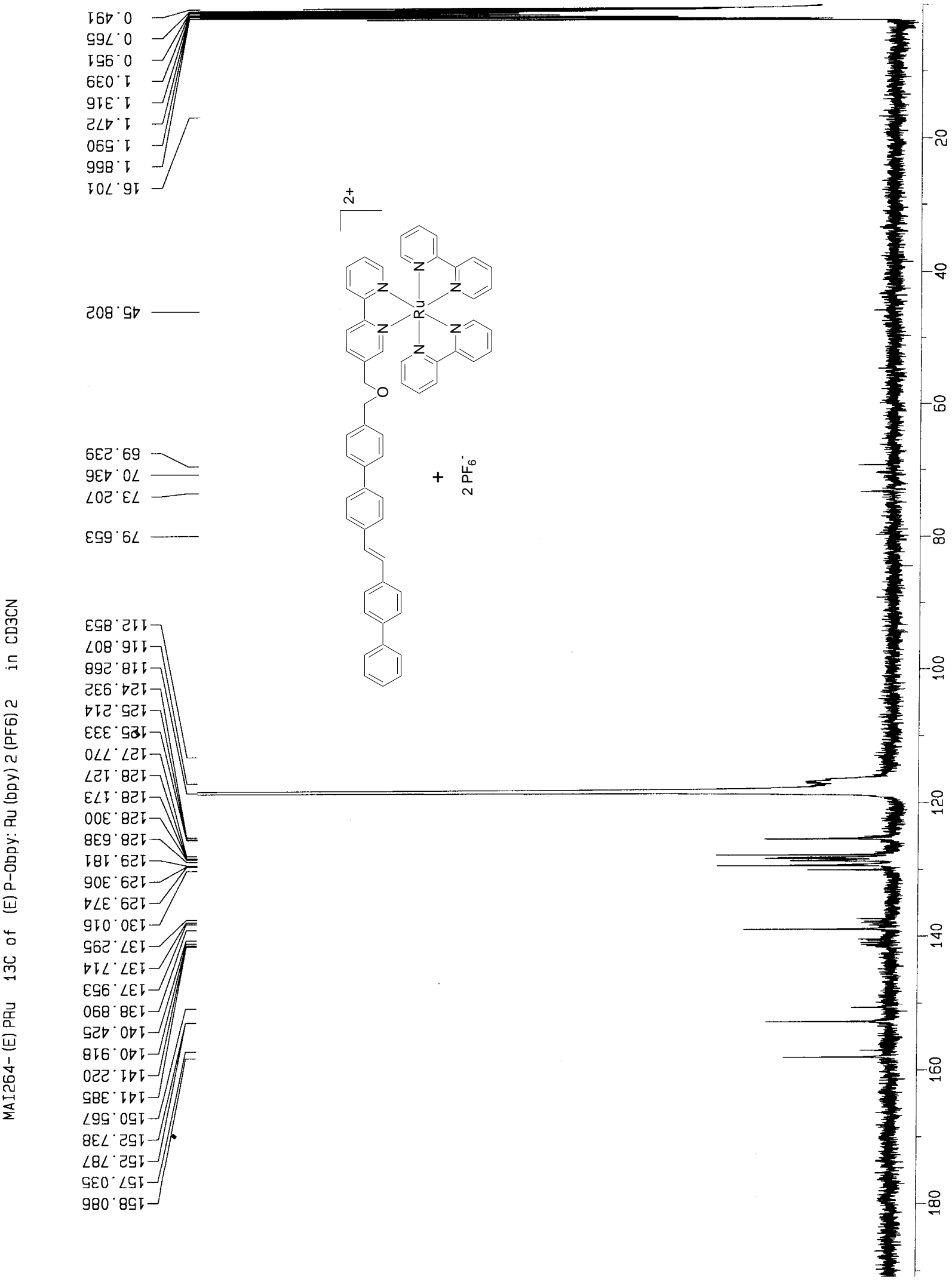


Ams, M., and Wilcox, C. S.. 